\title{
Optimal Scheduling of Hydrothermal System with Network and Ramping via SCE-UA Method
}

\author{
Jia-Rui Dong ${ }^{1}$, Chui-Yong Zheng ${ }^{*}{ }^{, 1,2}$, Guang-Yuan Kan $^{3}$, Min Zhao ${ }^{1,2}$ \\ ${ }^{1}$ Business School, Hohai University, Nanjing 210098, P.R. China \\ ${ }^{2}$ Water Resources and Sustainable Development Research Center of Jiangsu Province, Nanjing 210098, China \\ ${ }^{3}$ College of Hydrology and Water Resources, Hohai University, Nanjing, China
}

\begin{abstract}
This paper describes a method for scheduling large-scale hydrothermal power systems based on the shuffled complex evolution (SCE-UA) method. A multi-reservoir cascaded hydro-electric system with a non-linear relationship between water discharge rate, net head and power generation is considered. The water transport delay between connected reservoirs is also taken into account. SCE-UA is a successfully proven method in global optimization for many situations. Benefiting from its unique global optimization strategies into the inverse procedure greatly enhances the performance of SCE-UA method since it can not only effectively locate the promising areas in the solution space for a global minimum but also avoid its wandering near the global minimum in the final stage of search. The efficiency of the SCE-UA method is analyzed in terms of the mean performance and computational time, in comparison with the particle swarm optimization (PSO) algorithm. The simulation results reveal that SCE-UA effectively overcomes the premature phenomenon and improves the global convergence and optimization searching capability. It is a relatively consistent, effective and efficient optimization method in solving the large scale hydrothermal scheduling problem.
\end{abstract}

Keywords: Hydrothermal scheduling, Multichain reservoirs, penalty function, practical constraints, SCE-UA algorithm, valvepoint effects.

\section{INTRODUCTION}

The short-term hydro-thermal coordination problem (STHTC) is a crucial task in the economic operation of a power system. A good generation schedule reduces the production cost, increases the system reliability, and maximizes the energy capability of reservoirs by utilizing the limited water resource. The primary objective of the short term hydro thermal scheduling is to find the generation levels of the hydro and thermal units so as to minimize the fuel cost of thermal units. In classical optimization methods, the thermal input-output characteristics are approximated by a smooth differentiable or piecewise quadratic objective function. However, due to the valve-point effects, the real input-output characteristics contain higher order nonlinearity and discontinuity which results in a non-convex, non-smooth fuel cost function.

The STHTC is a nonlinear, high-dimensional, multiconstraint, complex water-machine electrical coupling optimization problem, which is constrained by cascaded hydro plants, water time delay, nonlinear hydro production function, release targets, power transmission limits [1]. Various optimization techniques have been applied to solve the STHTC problem. Applied optimization methods can be classical calculus-based algorithms such as linear and

*Address correspondence to this author at the Business School, Hohai University, Nanjing 210098, P.R. China; Tel: +86-25-83786327;

Fax: +86-25-83786319; E-mail: chyzheng@hhu.edu.cn nonlinear programming [2], interior point [3,4] and dynamic programming [5], Lagrange relaxation method [6,7]. The other methods are the artificial intelligence techniques including network flow method [8], heuristic methods, expert systems and artificial neural networks $[9,10]$. Orero and Irving present GA approach to STHTC of generators with prohibited operating zones and compared the results with lambda-iteration method and DP method [11]. Park et al. have developed PSO approach to solve economic dispatch problem and compared the results with improved evolutionary programming and modified Hopfield neural network [12]. Although these heuristic methods have been employed to solve the complex nonlinear, discrete STHTC problems, they do not always guarantee the globally optimal solution in finite time and is the difficulty of treating largescale systems. In order to reduce the dimension of the problem, aggregation of hydroelectric subsystem into an equivalent hydroplant is the common manipulation. Unfortunately, this practice is sometimes not adapted and unusable [13].

As a result of the above limitation, the Shuffled Complex Evolution (SCE-UA) which is capable of dealing with global optimization problem characterized by the large number of local minima, without the need of calculating any gradient or partial derivative information, especially for addressing problems for which the objective functions are not differentiable, stochastic, or even discontinuous [14]. The SCE-UA method is becoming much more useful and has 
recently been used to optimize complex mathematical problem of multiparameters, and multimodal objective functions. However, few attempts have been made to address the STHTC optimization problems.

In this paper, we implemented and tested STHTC problem based on the SCE-UA approach. The calculation efficiency and stability of the proposed method are tested on the hydro-thermal generation system with multi-chain hydro sub-system along with a number of thermal plants. The model considered takes into account non-linear cost function of thermal production, variations of water head in hydroelectric plants, non-linear function of hydroelectric output, a system of cascaded hydroelectric plants including dams with limited spillage capacity. A comparative analysis with Particle swarm optimization is also made in the present study to further evaluate the performance of the proposed approach. Results from both synthetic and actual field data demonstrate that SCE-UA algorithm applied to STHTC optimization problems should be considered robust, effective and efficient.

\section{PROBLEM FORMULATIONS}

\subsection{Notion}

To formulate the problem mathematically, the following notation used in this paper is first introduced:

$F_{T}:$ total production cost function

$F_{j}\left(P_{s j}^{t}\right):$ production cost for $P_{s j}^{t}$

$P_{s j}^{t}$ : power generation of thermal unit $\mathrm{j}$ at time interval $t$;

$P_{s j}^{\min }:$ minimum power generation of hydro plant $j$;

$P_{s j}^{\max }:$ maximum power generation of hydro plant $j$;

$N$ : number of thermal units

$T$ : number of time intervals

$M$ : number of hydro units

$P_{h i}^{t}$ : power generation of hydro unit $i$ at time interval $t$

$P_{h i}^{\min }:$ minimum power generation of hydro plant $i$;

$P_{h i}^{\max }$ :maximum power generation of hydro plant $i$;

$P_{D}^{t}$ : system load demand at time interval $t$;

$P_{L}^{t}$ : system total losses at time interval $t$

$a_{j}, b_{j}, c_{j}, e_{j}, f_{j}:$ cost curve coefficients of $j$ th thermal unit

$C_{1, i} \ldots C_{6, i}$ : power generation coefficients of the hydro unit $i$;

$V_{h i}^{t}$ :water volume of reservoir $i$ at the end of time interval $t$;

$V_{h i}^{\min }:$ minimum water volume of reservoir $i$;

$V_{h i}^{\max }:$ maximum water volume of reservoir $i$;

$V_{h i}^{i n i}:$ initial water volume of reservoir $i$;
$V_{h i}^{f i n}:$ final water volume of reservoir $i$;

$q_{h i}^{t}:$ water discharge for the hydro unit $i$ during interval $t$;

$q_{h i}^{\min }:$ minimum water discharge of hydro plant $i$;

$q_{h i}^{\max }$ : maximum water discharge of hydro plant $i$;

$r_{h i}^{t}$ : inflow rate into reservoir $i$ during time interval $t$;

$s_{h i}^{t}$ : spillage rate of the reservoir $i$ during interval $t$;

\subsection{Objective Function}

The prime objective of the STHTC problem is to minimize the total thermal cost such that all the equality and inequality operation constraints are satisfied, the load demands $P_{D}^{t}$ supplied from hydro and thermal plant in the intervals of generation scheduling can be met [15].

$\min F_{T}=\sum_{t=1}^{T} \sum_{j=1}^{N} F_{j}\left(P_{s j}^{t}\right)$

To obtain an accurate cost function model, the valvepoint effects are modeled in the fuel function as follow:

$F_{j}\left(P_{s j}^{t}\right)=a_{j}+b_{j} P_{s j}^{t}+c_{j}\left(P_{s j}^{t}\right)^{2}+\left|e_{j} \sin \left(f_{j}\left(P_{s j}^{\min }-P_{s j}^{t}\right)\right)\right|$

Subject to the following constraints:

1) The output hydro power is expressed as a function of the water discharge rate and the reservoir storage [15]:

$$
P_{h i}^{t}=C_{1, i}\left(V_{h i}^{t}\right)^{2}+C_{2, i}\left(q_{h i}^{t}\right)^{2}+C_{3, i} V_{h i}^{t} q_{h i}^{t}+C_{4, i} V_{h i}^{t}+C_{5, i} q_{h i}^{t}+C_{6, i}
$$

2) Active power balance constraints : this constraint is based on the principle of equilibrium between the total generation from hydro and thermal plants and the total system demand plus the system losses [16].

$$
\sum_{i=1}^{M} P_{h i}^{t}+\sum_{j=1}^{N} P_{s j}^{t}-P_{D}^{t}-P_{L}^{t}=0
$$

3) Thermal and hydro plant power generation limits: the operating limit of plant has a lower and upper bound so that it lies in between these bounds.

$$
\begin{aligned}
& P_{s j}^{\min } \leq P_{s j}^{t} \leq P_{s j}^{\max } \\
& P_{h i}^{\min } \leq P_{h i}^{t} \leq P_{h i}^{\max }
\end{aligned}
$$

Hydro plant discharge limits: the physical limitation of water discharge must lie in between its maximum and minimum operating limits.

$q_{h i}^{\min } \leq q_{h i}^{t} \leq q_{h i}^{\max }$

5) Reservoir storage volumes limits: the operation volume of reservoir storage limit must lie in between the minimum and maximum capacity limits.

$$
V_{h i}^{\min } \leq V_{h i}^{t} \leq V_{h i}^{\max }
$$


6) Water dynamic balance equation: the storage reservoir volume limits are expressed with given initial and final volumes as [17]:

$$
V_{h i}^{t}=V_{h i}^{t-1}+\left[r_{h i}^{t}-q_{h i}^{t}-s_{h i}^{t}\right]+\sum_{m=1}^{N_{u}}\left[q_{h m}^{t-\tau_{m, i}}+s_{h m}^{t-\tau_{m, i}}\right]
$$

7) Ramp rate constraints: the ramp rate limits are caused by the fact that the thermal generating output cannot be adjusted instantaneously. The operating range of all on-line is restricted by their ramp rate limits [18]:

$$
\left\{\begin{array}{l}
P_{s j}^{t} \geq \max \left(P_{s j}^{\min }, P_{s j}^{t-1}-\Delta P_{s j}^{d o w n}\right), P_{s j}^{t} \leq P_{s j}^{t-1} \\
P_{s j}^{t} \leq \min \left(P_{s j}^{\max }, P_{s j}^{t-1}+\Delta P_{s j}^{u p}\right), P_{s j}^{t} \geq P_{s j}^{t-1}
\end{array}\right.
$$

\section{SHUFFLED COMPLEX EVOLUTION (SCE-UA)}

The SCE-UA method is a new global optimization strategy which is good at effectively and effectively solving a broad class of problem. The SCE-UA strategy not only combines the concepts of controlled random search, competitive evolution, but also complex shuffling which is a newly developed sense [19]. Such combinations ensure that all the information comes from the sample can be thoroughly and quickly exploited. Thus it could be reasonably expect that SCE-UA method should have much better global convergence on solving a wider range of problems. It means if a series of function evaluation was given, the SCE-UA method could exhibit a higher probability in finding the global optimum towards its objective.

The essence of the method can be summarized as follow:

1) Beginning with a group of points which were randomly selected from the feasible space. Such group is divided into several communities. In each community, it contains $2 n+1$ points. Here $n$ is the dimension of the problem. 2) Each community is made to evolve using competitive complex evolution (CCE) techniques that uses the simplex geometric shape to direct search in an improvement direction. At periodic stages in the evolution, the entire population is shuffled and points are reassigned to communities to ensure information sharing. 3) As the search progresses, the entire population tends to converge toward the neighborhood of global optimum, provided the initial population size is sufficiently large [20].

The essential principles and theories for the SCE-UA method could be concluded briefly as follow: Each community can be seen as a potential parent and such parent community was subjected to a process of reproduction; a sub complex which consists of more than two members selected form the whole complex can be used as a pair of parents. Here, we should ensure that the probability which was contributed to the offspring from the better parents must higher than that of worse ones in order to make sure that this evolution process is competitive. Therefore the employment of a triangular probability distribution can realize the competitive evolution. For each sub complex which is used for the generation of most of the offspring, Nelder and
Mead's procedure is introduced. This combination can improve the evolution into a profitable direction by making use of the information derived from each sub complex [20]. Moreover, in order to discharge the unpromising regions and make the evolution not to get trapped, offspring are located randomly under certain conditions. This is somewhat analogous to biological evolution in which mutation was produced in response to the outside stimuli. Such mutation may increase the information stored in samples. Consequently, the obtained new offspring replaces the worst region in the sub complex, rather than in the whole population. In order that, each parent has at least one chance to be devoted into the reproduction step before being replaced or discarded. Therefore, all information contained in the sample will be effectively collected [21].

\section{SHUFFLED COMPLEX EVOLUTION ALGORITHM STEPS FOR HYDRO-THERMAL SCHEDULING}

In this section, the procedures of proposed SCE-UA for solving short-term generation scheduling of hydro system is described in details. Penalty function is proposed to handle the equality, inequality constraints especially active power balance constraint and ramp rate constraints. The process of the SCE-UA algorithm can be summarized as follows [22].

\subsection{Initialization}

To initialize the process, it is defined $p \geq 1$ and $m \geq n+1$, where $p$ represents the number of complexes, $m$ is the number of points contained by certain complex, and $n$ indicates the dimension for this problem. Compute the sample size $s=p m$.

\subsection{Generates a Sample}

In the initialization process, a set of individuals is created at random. The structure of an individual for STHTC problem is composed of a set of power output of thermal plant and discharge decision variables for each hydro plant in over the scheduling horizon. Each individual's position contains real numbers randomly generated.

$$
\text { Let } X_{s}=\left[P_{s 1}, P_{s 2}, \cdots, P_{s j}, \cdots, P_{s N}, q_{h 1}, q_{h 2}, \cdots, q_{h i}, \cdots, q_{h M}\right]
$$
be a trial matrix designating the $s$ th individual of a population to be evolved and $P_{s j}=\left[P_{s j}^{1}, P_{s j}^{2}, \cdots, P_{s j}^{t}, \cdots, P_{s j}^{T}\right], q_{h i}=\left[q_{h i}^{1}, q_{h i}^{2}, \cdots, q_{h i}^{t}, \cdots, q_{h i}^{T}\right]$ . The elements $P_{s j}^{t}$ and $q_{h i}^{t}$ are the power output of the $j$ th thermal unit and the discharge rate of the $i$ th hydro plant at time $t$. The range of the elements $P_{s j}^{t}$ and $q_{h i}^{t}$ should satisfy the thermal generating capacity and the water discharge rate constraints in (5) and (7), respectively.

To meet exactly the restrictions on the initial and final reservoir storage in (8), the water discharge rate of the $i$ th hydro plant $q_{h i}^{d}$ in the dependent interval $d$ is then calculated by

$$
q_{h i}^{d}=V_{h i}^{0}-V_{h i}^{T}+\sum_{t=1}^{T} r_{h i}^{t}+\sum_{t=1}^{T} \sum_{m=1}^{N_{u}}\left[q_{h m}^{t-\tau_{m, i}}+s_{h m}^{t-\tau_{m, i}}\right]-\sum_{\substack{t=1 \\ t \neq d}}^{T} q_{h i}^{t}-\sum_{t=1}^{T} s_{h i}^{t}
$$


Using these hydro discharges, the volumes at different intervals are determined. According to hydro plant generation characteristics, hydro plant generation power can be obtained using its hydro discharges and storage volumes. From the calculated hydro generation power, the thermal generation power is calculated using (12). The thermal generation $P_{s d_{g}}^{t}$ of the dependent thermal generating unit $d_{g}$ can then be calculated using the following equation:

$$
P_{s d_{g}}^{t}=P_{D}^{t}-\sum_{\substack{j=1 \\ j \neq d_{g}}}^{N_{s}} P_{s j}^{t}-\sum_{j=1}^{N_{h}} P_{h i}^{t}
$$

\subsection{Rank the Points}

Sample $s$ points to the ensemble of $x_{1}, \cdots, x_{s}$ in the feasible space. Compute the function value $F_{i}$ in equation (2) at each point $x_{i}$. Due to the absence of prior information, a uniform sampling distribution was chosen. Sort the $s$ points in order of increasing function value. Store them in an array $D=\left\{x_{i}, F_{i}, i=1, \cdots, s\right\}$, thus $i=1$ indicates the point exhibits the smallest function value.

\subsection{Partition into Complexes}

Ensemble $D$ was divided into $p$ complexes $A^{1}, \cdots, A^{p}$. Each complex contains $m$ points. Thus the first complex contains every $p(j-1)+1$ ranked point, while the second complex contains every ranked point of $p(j-1)+2$ of $D$, where $j=1,2, \ldots, m$.

\subsection{Evolve Each Sequence}

Based on the algorithm of competitive complex evolution (CCE), each of the parallel sequence was evolved [19].

\subsection{Shuffle the Complexes as Follows}

Ensemble $A^{1}, \cdots, A^{p}$ was replaced into $D$, obtained $D=\left\{A^{k}, k=1, \cdots, p\right\}$. Sort the ensemble of $D$ in the order of function value increasing.

\subsection{Check Convergence}

The convergence criteria is one of following conditions: 1) When the specified precision for target function cannot be further improved (eg. better than 0.01) after certain cycles. 2) the maximum iteration number. If the predefined maximum iteration number has been reached. If the convergence criteria are satisfied, stop; otherwise, return to section 4.4. The competitive complex evolution (CCE) algorithm is presented in following scheme [22]:

Step 1: Initialization of the process.

$\alpha$ : The number of offspring which each subcomplex generates before it is put back into complex. It can be any number greater than or equal to one. As $\alpha$ is increased, the search biased in factor of local search becomes more strongly.

$\beta$ : The number of evolution steps taken by each complex before the complexes are shuffled. If $\beta$ is large, each complex will shrink into the small cluster and lost its global search effectiveness. If $\beta$ is small, the complexes will not conduct much independent exploration of the parameter space.

Step 2: Assignation. The point $x_{1}^{k}$ exhibits the highest probability $\rho_{1}=\frac{2}{m+1}$, while the point $x_{m}^{k}$ with the lowest

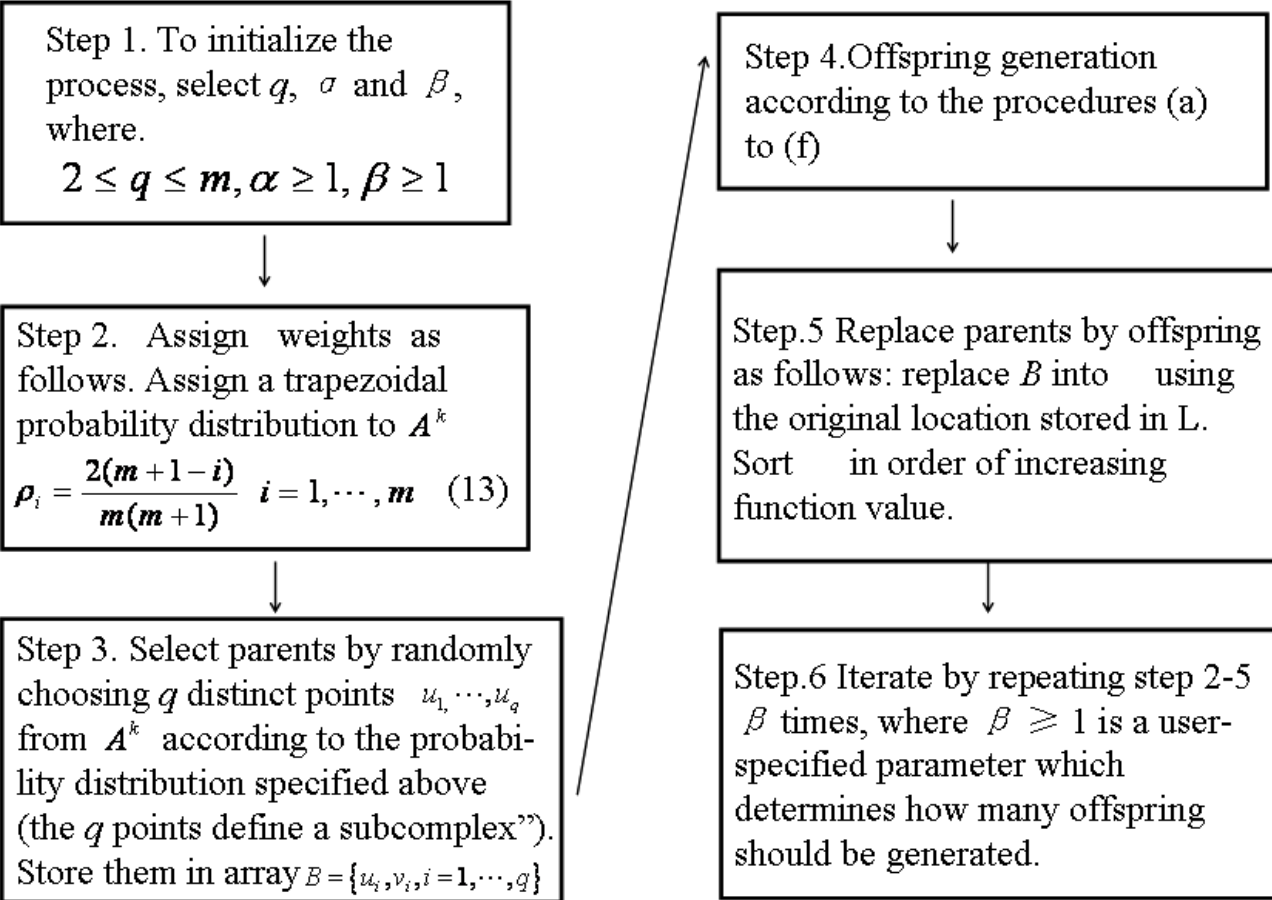

Step 1. To initialize the process, select $q, a$ and $\beta$, where.

Step 2. Assign weights as follows. Assign a trapezoidal probability distribution to $A^{k}$ $\rho_{i}=\frac{2(m+1-i)}{m(m+1)} \quad i=1, \cdots, m$ (13) Store them in array $B=\left\{u_{i}, v_{i}, i=1, \cdots, q\right\}$

Scheme 1. the procedure of competitive complex evolution (CCE). 
probability $\rho_{m}=\frac{2}{m(m+1)}$.

Step 3: Parents selection. $v_{i}$ is the function value, which is associated with point $u_{i} . u_{i}$ was stored in $\mathrm{L} . \mathrm{L}$ is the location of $A^{k}$ used to construct B.

Step 4: Generate offspring according to the following procedure: (a) Sort B and L so that the $q$ points are arranged in order of increasing function value and compute the centroid g using the expression:

$$
g=\frac{1}{q-1} \sum_{j=1}^{q-1} u_{j}
$$

(b) compute the new point $r=2 g-u_{g}$. (c) If $\mathrm{r}$ is within the feasible space, compute the function value $f_{r}$ and go to step $\mathrm{d}$; otherwise compute the smallest hypercube $H$ that contains $A^{k}$, randomly generate the point $z$ within $H$, compute $f_{z}$, set $r=z$ and $f_{r}=f_{z}$. (d) If $f_{r}<f_{q}$, replace $u_{q}$ by $r$, go to step f; otherwise compute $c=\left(g+u_{q}\right) / 2$ and $f_{c}$. (e) If $f_{c}<f_{q}$, replace $u_{q}$ by $c$, go to step $\mathrm{f}$; otherwise randomly generate a point $z$ within $H$ and compute $f_{z}$. Replace $u_{q}$ by $z$. (f) Repeat steps ae $\alpha$ times, where $\alpha \geq 1$ is a user-specified parameter.

Step 5: Replacement of parents by offspring.

Step 6: Iteration

\section{SIMULATION RESULT}

\subsection{Test System}

In this section, the test system is studied to demonstrate the feasibility and effectiveness of the proposed SCE-UA method for solving STHTC. A 6-unit test system with fuel cost function takes into account the valve-point effects to demonstrate the performance of SCE-UA method. Normally, short term hydro thermal scheduling concerns with one day to week periods of operation with interval of various lengths. This paper focuses on short term hydro-thermal scheduling, in which day ahead scheduling is done for 24hours on 2 hours time interval. The test system comprises of a multi-chain cascade of three hydro plants and three thermal plants. The configuration of the hydraulic sub-system is shown in Fig. (1). The hydro power generation coefficients, reservoir limits are given in Tables $\mathbf{1}$ and $\mathbf{2}$, respectively. The generation limits, cost coefficients of thermal units are given in Table $\mathbf{3}$.

\subsection{Optimal Estimation of the SCE-UA Parameters of STHTC Problem}

Before proceeding to the simulated calculation, careful selection of parameter setting is important to produce a competent result. Selection of the parameters considerably affects the performance of the SCE-UA for STHTC problem. The SCE-UA method contains many probabilistic and deterministic components that are controlled by some algorithmic parameters. The complex size of $m$ is chosen to be equal to $2 n+1$. Here $n$ represents the dimension of the problem. $\beta$ indicates the number of offspring, which can be generated by each independently evolving complex. Such complex is between two consecutive shuffles which also has the same complex size $(2 n+1)$. Concerning about the subcomplex selected for generation, its size of was chosen to be $n+1$, which is the standard size for a simplex. Such definition was also specified by Nelder and Mead that

Table 1. Hydroelectric Plant Coefficients

\begin{tabular}{|c|c|c|c|c|c|c|}
\hline & $\boldsymbol{C}_{\boldsymbol{I}}$ & $\boldsymbol{C}_{\mathbf{2}}$ & $\boldsymbol{C}_{\mathbf{3}}$ & $\boldsymbol{C}_{\boldsymbol{4}}$ & $\boldsymbol{C}_{\boldsymbol{5}}$ & $\boldsymbol{C}_{\boldsymbol{6}}$ \\
\hline \hline Hydro 1 & -0.0029 & -0.31 & 0.03 & 1.34 & 14 & -70 \\
\hline Hydro 2 & -0.0032 & -0.3 & 0.04 & 1.14 & 23 & -55 \\
\hline Hydro 3 & -0.003 & -0.21 & 0.027 & 1.44 & 11.5 & -80 \\
\hline
\end{tabular}

Table 2. Hydroelectric Plant Data

\begin{tabular}{|l|c|c|c|c|c|c|c|c|}
\hline & $V_{h i}^{\min }\left(10^{4} \mathrm{~m}^{3}\right)$ & $V_{h i}^{\max }\left(10^{4} \mathrm{~m}^{3}\right)$ & $V_{h i}^{i n i}\left(10^{4} \mathrm{~m}^{3}\right)$ & $V_{h i}^{f i n}\left(10^{4} \mathrm{~m}^{3}\right)$ & $q_{h i}^{\min }\left(10^{4} \mathrm{~m}^{3} / \mathrm{s}\right)$ & $q_{h i}^{\max }\left(10^{4} \mathrm{~m}^{3} / \mathrm{s}\right)$ & $P_{h i}^{\min }(\mathrm{MW})$ & $P_{h i}^{\max }(\mathrm{MW})$ \\
\hline \hline Hydro 1 & 80 & 150 & 88 & 120 & 5 & 25 & 0 \\
\hline Hydro 2 & 60 & 130 & 70 & 80 & 6 & 25 & 0 \\
\hline Hydro 3 & 30 & 120 & 60 & 110 & 10 & 40 & 0 \\
\hline
\end{tabular}

Table 3. Cost Curve Coefficients and Limits of Thermal Generations

\begin{tabular}{|c|c|c|c|c|c|c|c|}
\hline & $a_{j}$ & $b_{j}$ & $c_{j}$ & $e_{j}$ & $f_{j}$ & $P_{s j}^{\min }(\mathbf{M W})$ & $P_{s j}^{\max }(\mathbf{M W})$ \\
\hline \hline Hydro 1 & 100 & 2.45 & 0.0012 & 160 & 0.0038 & 20 & 175 \\
\hline Hydro 2 & 120 & 2.32 & 0.0010 & 180 & 0.0027 & 40 & 300 \\
\hline Hydro 3 & 150 & 2.1 & 0.0015 & 200 & 0.0035 & 50 & 500 \\
\hline
\end{tabular}




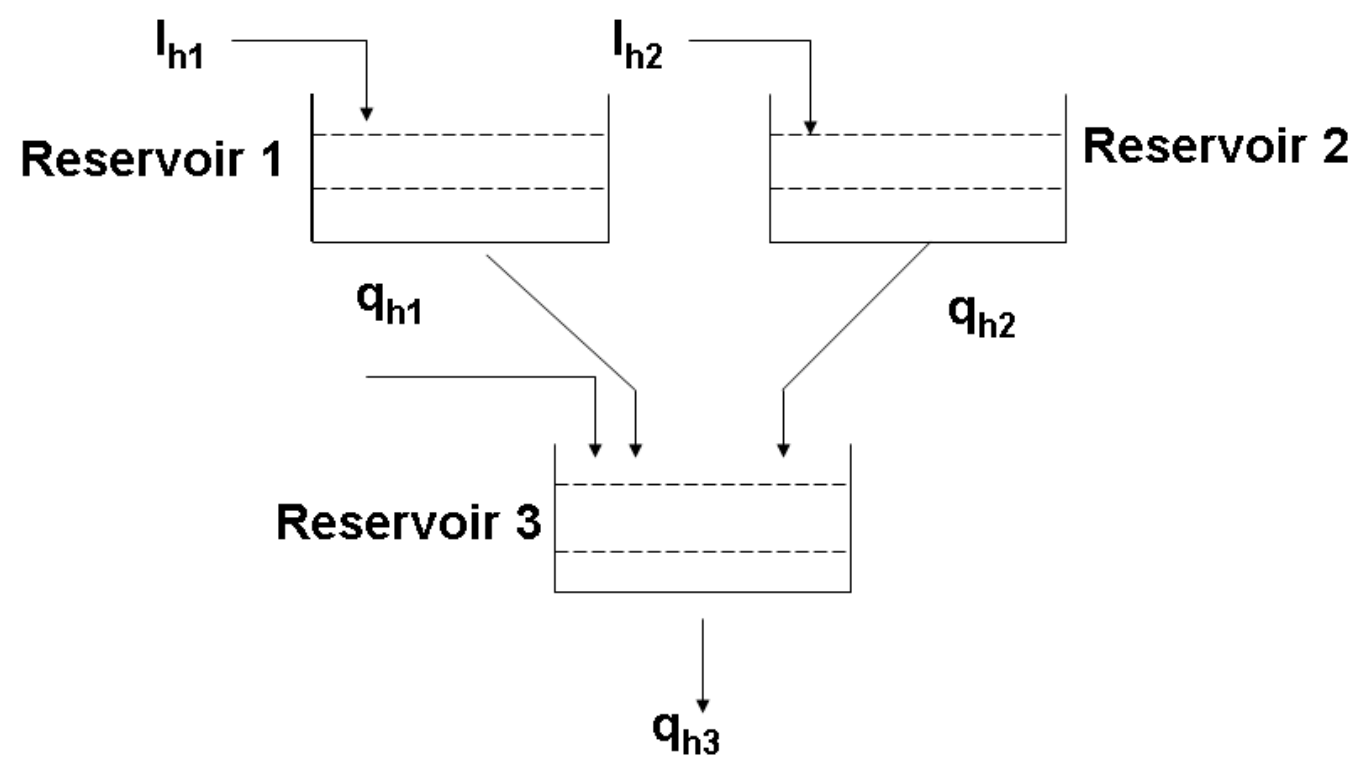

\section{$I_{\text {hi }}$ : natural inflow to $i^{\text {th }}$ reservoir}

\section{$\mathrm{q}_{\mathrm{hi}}$ :discharge of $\mathrm{i}^{\text {th }}$ plant}

Fig. (1). The configuration of hydro sub-system.

employed in the CRS procedure reported in previous study [20]. The parameter $\alpha$ was set to 1 in order to match the CRS procedure. In this method, $p$, the number of complexes was selected and endowed to the primary variable.

To successfully implement the SCE-UA, the values of key parameter $p$ have to be determined first. In order to find optimal values of $p$ to solve the STHTC problem considered in this paper, experiments are conducted using the proposed method by increasing $p$ from 10 to 20 in steps of 1 . For each value for this parameters $p, 20$ independent trials have been made to bring out the optimal values $p$ for the proposed SCE-UA to solve STHTC problem. The results of case, for worst, average and best total fuel costs obtained by SCE-UA for each parameters $p$ are shown in Table 4. Based on the results from Table 4 , the proposed SCE-UA method provides the best results when $p=17$.

\subsection{Computation Results}

Based on the statistics outcome of experiments shown in Table 4, parameters chosen in this paper are as follows: the number of complexes $p=17$; maximum number of function evaluations allowed during optimization $\operatorname{maxn}=1 \mathrm{e} 5$; maximum number of evolution loops before convergence $k s t o p=10$, the percentage change allowed in kstop loops before convergence percento $=0.01$.

In order to check solution quality, we inspected the variation in total fuel cost from 20 trials using the proposed SCE-UA and PSO methods. The hydrothermal generation scheduling and the optimal hydro discharge with minimum cost obtained by the SCE-UA and PSO method are reported in Tables $\mathbf{5}$ and $\mathbf{6}$ respectively. In Table 5, the last column provides sum of power generation for all generators in order to check whether the total load balance constrains are satisfied or not. The solution for SCE-UA method is optimal and also satisfies the total load balance constraints completely for short-term hydro generation scheduling, while PSO algorithm is not satisfied. Fig. (2) is peak-shaved load curve scenario with SCE-UA and PSO methods. Fig. (3) is the hydro power generation obtained by SCE-UA. From Fig. (2), it is clearly seen that each thermal plant load generates variation in a small range with trial numbers using the SCE-UA method, thus verifying that the proposed SCEUA has better quality of solution to ensure the operation of thermal plants continuously and reducing the number of switch machine. As seen in the simulation results of the test system, the schedule of thermal plants obtained by SCE-UA method is suitable to operate at base load plants, leaving

Table 4. SCE-UA Parameter Tuning Results

\begin{tabular}{|c|c|c|c|c|c|c|c|c|c|c|c|}
\hline & 10 & 11 & 12 & 13 & 14 & 15 & 16 & 17 & 18 & 19 & 20 \\
\hline Max & 15063.28 & 15177.36 & 15103.85 & 15328.44 & 14924.72 & 15174.42 & 14927.38 & 14879.35 & 15407.39 & 15182.54 & 15358.86 \\
\hline Ave & 14901.83 & 14973.66 & 14973.82 & 15125.60 & 14767.41 & 14889.54 & 14798.04 & 14744.97 & 15139.14 & 14862.64 & 14945.65 \\
\hline Min & 14876.38 & 14910.32 & 14894.82 & 19837.59 & 14689.50 & 14818.48 & 14707.71 & 14672.94 & 14937.86 & 14788.94 & 14855.75 \\
\hline
\end{tabular}


hydro-plants to operate at peak load plants. It reduces the production cost, increases the system reliability, and maximizes the energy capability of reservoirs by utilizing the limited water resource.

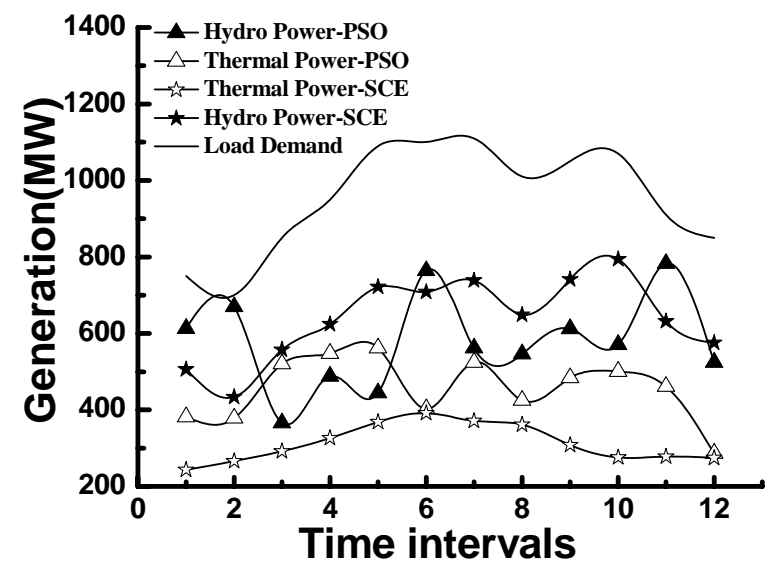

Fig. (2). Peak-shaved load curve scenario.

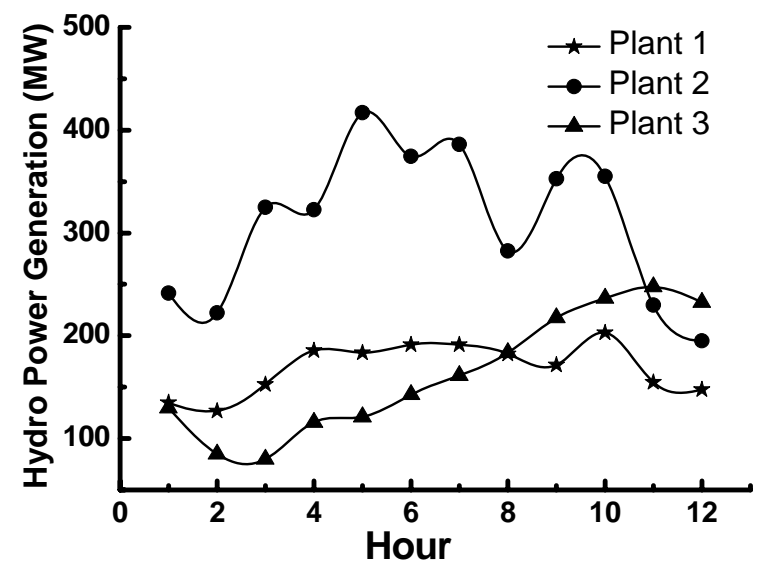

Fig. (3). Hydro power generation with SCE-UA.

In order to check solution quality, we inspected the variation in total thermal plant cost and its standard and standard deviation from 20 trials. Table 7 provides

Table 5. Hydrothermal Generation Schedule by SCE-UA and PSO

\begin{tabular}{|c|c|c|c|c|c|c|c|c|c|c|c|c|c|}
\hline \multicolumn{7}{|c|}{ SCE-UA (MW) } & \multicolumn{6}{|c|}{ PSO (MW) } & \multirow[b]{2}{*}{$P_{D}$} \\
\hline Hour & $P_{h 1}$ & $P_{h 2}$ & $P_{h 3}$ & $P_{s 1}$ & $P_{s 2}$ & $P_{s 3}$ & $P_{h 1}$ & $P_{h 2}$ & $P_{h 3}$ & $P_{s 1}$ & $P_{s 2}$ & $P_{s 3}$ & \\
\hline 1 & 135.06 & 241.32 & 129.85 & 49.82 & 106.89 & 87.07 & 109.12 & 352.57 & 151.39 & 42.01 & 226.47 & 113.11 & 750 \\
\hline 2 & 126.93 & 222.11 & 84.98 & 47.54 & 120.63 & 97.85 & 202.96 & 343.68 & 122.85 & 124.22 & 167.98 & 85.95 & 700 \\
\hline 3 & 152.59 & 324.98 & 80.07 & 66.75 & 121.67 & 103.83 & 110.59 & 172.53 & 82.57 & 145.44 & 207.75 & 167.30 & 850 \\
\hline 4 & 185.72 & 322.45 & 115.77 & 82.00 & 134.23 & 109.82 & 145.21 & 297.08 & 45.37 & 142.58 & 81.52 & 323.44 & 950 \\
\hline 5 & 183.54 & 416.84 & 121.13 & 94.77 & 152.10 & 121.56 & 226.87 & 157.70 & 60.07 & 131.98 & 102.48 & 327.04 & 1090 \\
\hline 6 & 191.43 & 374.33 & 142.66 & 98.66 & 160.59 & 132.35 & 214.93 & 453.89 & 95.21 & 123.57 & 184.22 & 94.82 & 1100 \\
\hline 7 & 191.27 & 386.01 & 161.45 & 84.55 & 155.01 & 131.62 & 150.12 & 365.22 & 46.22 & 104.59 & 184.65 & 234.86 & 1110 \\
\hline 8 & 182.43 & 282.40 & 183.58 & 82.00 & 155.08 & 124.46 & 139.57 & 327.52 & 78.74 & 77.10 & 111.32 & 236.06 & 1010 \\
\hline 9 & 171.58 & 352.58 & 217.44 & 65.82 & 137.18 & 105.38 & 201.48 & 314.34 & 96.36 & 45.60 & 235.14 & 203.28 & 1050 \\
\hline 10 & 203.06 & 354.84 & 236.37 & 70.13 & 119.72 & 85.89 & 142.32 & 285.47 & 142.68 & 110.08 & 118.31 & 272.02 & 1070 \\
\hline 11 & 154.72 & 229.93 & 247.40 & 72.55 & 110.21 & 95.16 & 203.93 & 426.42 & 152.13 & 172.66 & 209.82 & 79.02 & 910 \\
\hline 12 & 147.61 & 195.17 & 232.56 & 68.38 & 108.97 & 97.25 & 129.85 & 202.14 & 192.15 & 49.55 & 154.38 & 82.36 & 850 \\
\hline
\end{tabular}

Table 6. Hourly Plant Discharge $\left(\times 10^{4} \mathrm{~m}^{3}\right)$ Using SCE-UA and PSO

\begin{tabular}{|c|c|c|c|c|c|c|c|c|c|c|c|c|c|}
\hline Method & Hour & $\mathbf{1}$ & $\mathbf{2}$ & $\mathbf{3}$ & $\mathbf{4}$ & $\mathbf{5}$ & $\mathbf{6}$ & $\mathbf{7}$ & $\mathbf{8}$ & $\mathbf{9}$ & $\mathbf{1 0}$ & $\mathbf{1 1}$ & $\mathbf{1 2}$ \\
\hline \hline SCE-UA & $q_{h 1}^{t}$ & 8.16 & 7.41 & 9.55 & 13.02 & 12.41 & 12.72 & 12.21 & 10.95 & 9.69 & 12.95 & 8.15 & 7.44 \\
\hline SCE-UA & $q_{h 2}^{t}$ & 10.53 & 9.29 & 14.91 & 14.66 & 21.30 & 18.03 & 19.03 & 12.24 & 15.89 & 15.78 & 8.92 & 7.151 \\
\hline SCE-UA & $q_{h 3}^{t}$ & 15.28 & 10.07 & 10.00 & 18.21 & 16.98 & 21.17 & 21.54 & 22.23 & 24.87 & 25.64 & 22.67 & 17.70 \\
\hline PSO & $q_{h 1}^{t}$ & 5.83 & 17.10 & 6.31 & 8.86 & 24.46 & 22.96 & 10.44 & 8.83 & 17.09 & 9.35 & 19.93 & 9.34 \\
\hline PSO & $q_{h 2}^{t}$ & 17.62 & 17.36 & 7.32 & 13.67 & 6 & 23.66 & 17.16 & 14.59 & 13.33 & 11.38 & 20.20 & 7.55 \\
\hline PSO & $q_{h 3}^{t}$ & 21.93 & 25.99 & 26.6 & 21.38 & 21.60 & 19.68 & 38.48 & 20.14 & 15.54 & 39.14 & 16.98 & 28.19 \\
\hline
\end{tabular}


comparison of the minimum, maximum, average total fuel cost obtained from the proposed SCE-UA method with of PSO. From Table 7, the SCE-UA presents a better total fuel cost, and a lesser CPU time. In the paper, penalty function has been adopted to tackle and optimize the constraints of STHTC problem. If the calculation results do not meet the constraints, penalty function value should be greater than zero. Inversely, when the calculated results satisfy the constraints well, obtained value of the penalty function should be equal to zero. From Table 8, the penalty function value obtained by SCE-UA equal to zero. PSO method cannot satisfy the water dynamic balance limit, thermal \& hydro power generation limits and even water dynamic balance limit, thus, results obtained by PSO is greater than zero. The bigger value for penalty function obtain means the calculated results are far unable to meet the constraints.

Fig. (4) is the trajectories of reservoir storage volumes for STHTC obtained by SCE-UA. Fig. (5) shows the distribution of the best total thermal plant cost of each trial from running 20 times using the proposed SCE-UA and PSO methods. From Fig. (5), the convergence and the variation obtained by SCE-UA is tolerable. Average cost of 20 trials is very near the middle position that lies between their maximum and minimum values. The total fuel cost generates variation in a small range with trial number using the proposed SCE-UA method. It verifies that the proposed SCE-UA method enhances the efficiency of solving and reduces the probability of being trapped in local optimization.

In the meantime, we examine the variation in the total fuel cost of test system with evolutionary generation numbers, which illustrate the improvement achieved in the convergence property of the SCE-UA method compared with PSO. Figs. $(\mathbf{6}, 7)$ show the convergence process obtained by SCE-UA and PSO method. For the PSO method, there is a sharp decline in the total thermal plant cost at the beginning evolutionary stages until generation 40 while it declines slowly during later stages and the corresponding value is far away from the optimal solution. The thermal plant cost obtained by SCE-UA method decline much slowly than PSO in the entire evolutionary process. It leads to thermal cost smaller than PSO and can find the optimal

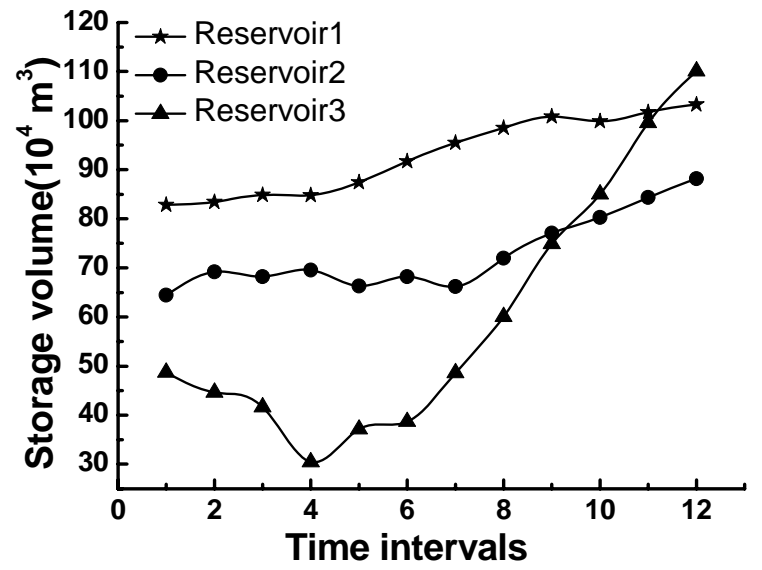

Fig. (4). Reservoir storage volumes obtained by SCE-UA.

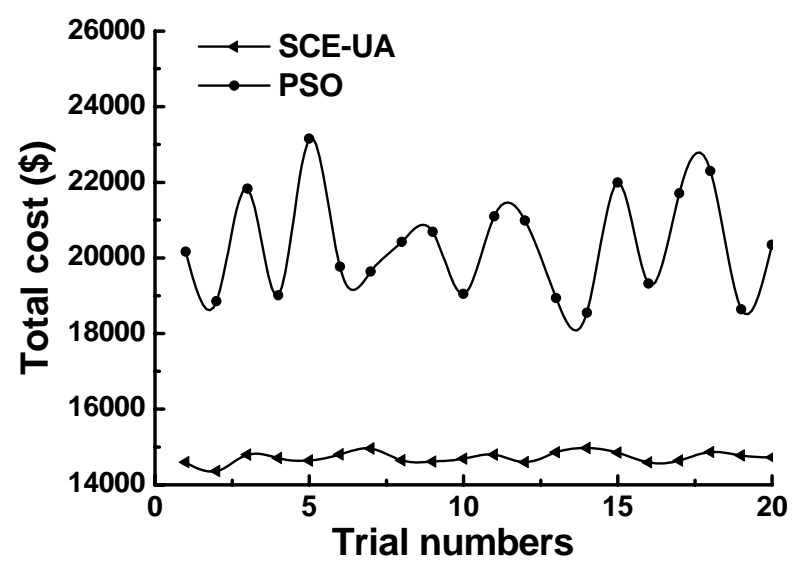

Fig. (5). Distribution of the best cost of each trial.

solution in the given maximum evolutionary iterative numbers and there is a large reduction in computation time. It shows that the convergence property of SCE-UA method is better than that of PSO for solving STHTC problem.

\section{CONCLUSIONS}

This paper has investigated the consistency with which global optimization algorithm is able to find the optimal

Table 7. Results (20 Runs) Obtained by Optimization Method

\begin{tabular}{|c|c|c|c|c|}
\hline Algorithm & Min.cost (\$) & Max.cost (\$) & Mean.coat (\$) & CPU Time (min) \\
\hline \hline SCE-UA & 14358.46 & 14971.60 & 14722.56 & 2 \\
\hline PSO & 18549.19 & 21991.67 & 20322.36 & 16 \\
\hline
\end{tabular}

Table 8. Results Obtained by Objective Constraints

\begin{tabular}{|c|c|c|c|c|c|}
\hline Method & $\begin{array}{c}\text { Thermal \& Hydro } \\
\text { Power Generation }\end{array}$ & $\begin{array}{c}\text { Hydro Plant } \\
\text { Discharge Limits }\end{array}$ & $\begin{array}{c}\text { Reservoir Storage } \\
\text { Volumes }\end{array}$ & $\begin{array}{c}\text { Water Dynamic } \\
\text { Balance }\end{array}$ & $\begin{array}{c}\text { Ramp } \\
\text { Rate }\end{array}$ \\
\hline \hline SCE-UA & 0 & 0 & 0 & 0 & 0 \\
\hline PSO & 373 & 0 & 0 & $8.94 \times 10^{10}$ & 371.357 \\
\hline
\end{tabular}




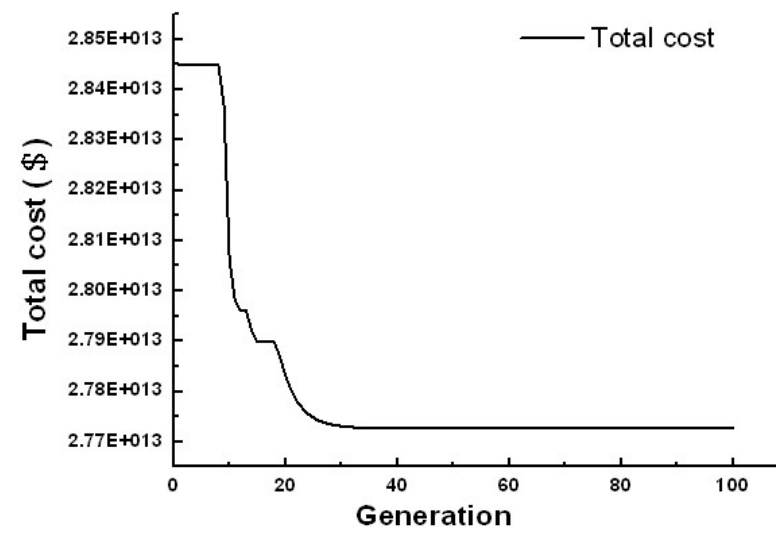

Fig. (6). Convergence process obtained by PSO.

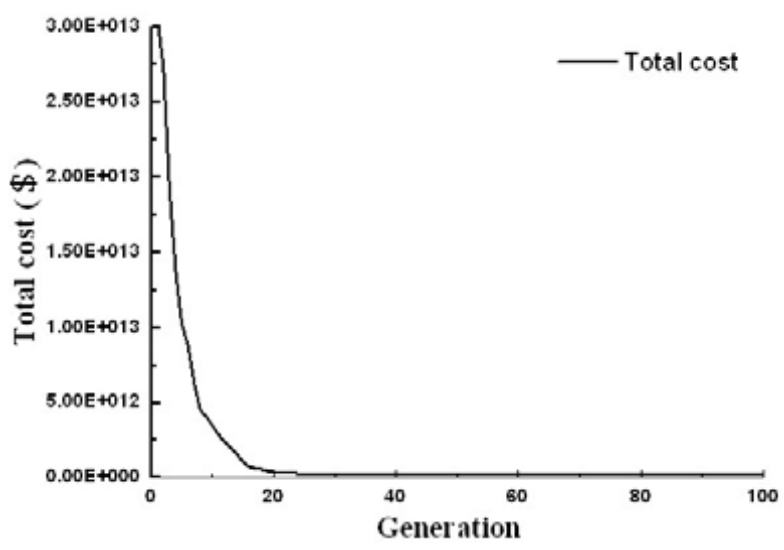

Fig. (7). Convergence process obtained by SCE-UA.

parameter values during calculating the hypothermal scheduling model. In the synthetic data study, SCE-UA effectively overcomes the premature phenomenon and improves the global convergence and global optimization searching capability, while the PSO method cannot exactly locate the global optimum. The simulation results of the case studies reveal that the SCE-UA algorithm can guide the search effectively under the help of obtained response surface information by using conclusive strategies. The selection of random elements in certain complicated complex makes such algorithm more flexible and generable. This strategy of systematic complex evolution ensures that the search is relatively targeted and very robust due to the guidance by the objective function. In order to handle constraints effectively, the penalty function is proposed to handle ramp rate constraint, reservoir volume constraints and active power balance constraints.

We have shown that the SCE-UA algorithm is a relatively consistent, effective and efficient optimization method to solve the short-term hydrothermal scheduling problem, which could reduce the total fuel cost, increases the system reliability and maximizes the energy capability of reservoirs by utilizing the limited water resource. Hence, an effective method is provided to solve the optimal daily generation scheduling of hydrothermal systems and it can be extended for applications in large-scale hydrothermal power systems.

\section{CONFLICT OF INTEREST}

The authors confirm that this article content has no conflict of interest.

\section{ACKNOWLEDGEMENTS}

This work was supported by the Key Projects in the National Science \& Technology Pillar Program (Grant No.2012BAB03B00)

\section{REFERENCES}

[1] A. Arce, T. Ohishi, and S. Soares, "Optimal dispatch of generating units of the Itaipu hydroelectric plant", IEEE. Trans. Power Syst., vol. 17, pp. 154-158, 2002.

[2] H. Habibollahzadeh, and G.X. Luo, "Hydrothermal optima power flow based on a combined linear and nonlinear programming methodology", IEEE. Trans. Power Syst., vol. 4, pp. 530-537, May 1989.

[3] J.P.S Catalao, H.M.I. Pousinho, and V.M.F. Mendes, "Mixedinteger nonlinear approach for the optimal scheduling of a headdependent hydro chain", Electr. Power Syst. Res., vol. 80, pp. 935$942,2010$.

[4] A.T. Anibal, A.R.L. Aurelio, and S. Soares, "Interior point method for long-term generation scheduling of large-scale hydrothermal systems", Ann. Oper. Res., vol. 169, pp. 55-80, 2008.

[5] C. Shi-Chung, C-H. Chen, I-K. Fong, and P.B. Luch, "Hydroelectric generation scheduling with an effective differential dynamic programming algorithm", IEEE. Trans. Power Syst., vol. 5, pp. 737-743, 1990.

[6] J.M. Ngundam, F. Kenfack, and T.T.Tatietse, "Optimal scheduling of large-scale of large-scale hydrothermal power systems using the Lagrangian relaxation technique", Int. J. Elect. Power, vol. 22, pp. 237-245, 2000.

[7] V.N. Dieu, and W. Ongsakul, "Enhanced merit order and augmented Lagrange Hopfield network for hydrothermal scheduling", Int. J. Elect. Power, vol. 30, pp. 93-101, 2008.

[8] G.G. Oliveira, "A second order network flow algorithm for hydrothermal scheduling", IEEE. Trans. Power Syst., vol. 10, pp. 1635-1641, 1995.

[9] R.H. Liang, and Y.Y.Hsu, "Scheduling of hydroelectric generation units using artificial neural networks", Proc. IEE, Pt. C, vol. 141, pp. 452-458, 1994.

[10] S. Soares, and T. Ohishi, "Hydro dominated short-term hydrothermal scheduling via a hybrid simulation-optimization approach: a case study." Proc. IEEE. Pt. C, vol. 142, pp. 569-575, Nov. 1995.

[11] S.O. Orero, and M.R. Irving, "Economic dispatch of generators with prohibited operating zone: a genetic algorithm approach", IEEE Proc. Gen. Transm. Distrib., vol. 143, pp. 529-34, 1996.

[12] P. Jong-Bac, L. Ki-Song, S. Joong-Rin, and Y. Kwang, "Ecomomic load dispatch for non-smooth cost function using particle swarm optimization". Power Eng. Soc. Gen. Meeting. IEEE, vol. 2, pp. 938-43, 2003.

[13] J.M Ngundam, F. Kenfack, and T.T. Tatietse, "Optimal scheduling of large-scale hydrothermal power systems using the Lagrangian relaxation technique", Int. J. Elect. Power, vol. 22, pp. 237-245, 2000.

[14] M. Nitin, and A.W. Jayawardena, "Shuffled complex evolution model calibrating algorithm enhancing its robustness and efficiency”. Hydrol. Process., vol. 22, pp. 4628-4638, 2008.

[15] P.K. Hota, A.K. Barisal, and R. Chakrabarti, "An improved PSO technique for short-term optimal hydrothermal scheduling", Electr. Power Syst. Res., vol.79, pp.1047-1053, 2009.

[16] Y. Xiaohui, S. Anjun, Y. Yanbin, N. Hao, and L. Wang, "An improved PSO for dynamic load dispatch of generators with valvepoint effects", Energy, vol.34, pp.67-74, 2009.

[17] K.K. Mandal, M. Basu, and N. Chakraborty, "Particle swarm optimization technique based short-term hydrothermal scheduling" Appl. Softw. Comput., vol.8, pp.1392-1399, 2008.

[18] L. Songfeng, S. Chengfu, and L. Zhengding, "An improved quantum-behaved particle swarm optimization method for short- 
term combined economic emission hydrothermal scheduling" Energy. Convers. Manage., vol.51, pp. 561-571, 2010.

[19] Q. Duan, S. Sorooshian, V.K. Gupta. "Optimal use of the SCE-UA global optimization method for calibrating watershed models". $J$. Hydro., vol. 158, pp. 265-284, 1994.

[20] V.C. Mariani, and L.S. Coelho, "A hybrid shuffled complex evolution approach with pattern search for unconstrained optimization”. J. Optim. Theory. Appl., vol. 81, pp. 1901-1909, 2011.

[21] Q.Y. Duan, V.K. Gupta, and S.Sorooshian, "Shuffled complex evolution approach for effective and efficient global minimization", J. Optim. Theory Appl., vol. 176, pp. 501-507, Mar. 1993.

[22] Q. Duan, S. Sorooshian, and V.K. Gupta, "Effective and efficient global optimization for conceptual rainfall- runoff models", Water Resour. Res., vol. 28, pp. 1015-1031, 1992.

(C) Dong et al.; Licensee Bentham Open.

This is an open access article licensed under the terms of the Creative Commons Attribution Non-Commercial License (http://creativecommons.org/licenses/by-nc/3.0/) which permits unrestricted, non-commercial use, distribution and reproduction in any medium, provided the work is properly cited. 